\title{
Apropiación de tecnologías en bibliotecas populares del Gran La Plata: Facebook desde la perspectiva del bibliotecario
}

\author{
Technology appropriation in Community Libraries from Gran La Plata: uses of Facebook from the librarian's \\ perspective
}

\author{
Alejandra Aracri \\ Universidad Nacional de La Plata. Facultad de Humanidades y Ciencias de la Educación - \\ Instituto de Investigaciones en Humanidades y Ciencias Sociales (CONICET-UNLP), \\ Argentina \\ alejandraaracri@gmail.com
}

\begin{abstract}
RESUMEN:
El presente artículo aborda la discusión de los usos de Facebook en las bibliotecas populares del Gran La Plata -Argentina- desde la perspectiva de la apropiación de tecnologías. Sobre un relevamiento de perfiles/páginas activos, se seleccionaron casos para una muestra no intencional, luego se exploró la apropiación de la herramienta que hacen los bibliotecarios y las bibliotecarias mediante entrevistas semiestructuradas. Los resultados de estas observaciones se articularon con la revisión de las recomendaciones de la bibliografía del campo bibliotecario. Se concluye que los usos de Facebook en las bibliotecas populares, si bien no se basan en la planificación que sugiere la bibliografía, se asientan sobre criterios tácitos construidos en base a la interpretación que realizan las y los bibliotecarios de la plataforma.
\end{abstract}

Palabras clave: Bibliotecas populares, Argentina, Facebook, Redes sociales, Apropiación de tecnologías.

\section{Abstract:}

This work addresses the discussion about uses of Facebook by the popular libraries of Gran La Plata -Argentina- from the perspective of a appropriation of the technology. On a survey of active profiles/pages, (there where selected cases for a unintentional sample); then, through interviews was explored the appropriation of the tool made by librarians. The results of these observations were articulated with the analysis with the review of the recommendations of the bibliography in the library field. The work concluded that the uses of Facebook in popular libraries are not based on the planning suggested by the bibliography, they based tacit criteria constructed on the interpretation of the platform by the librarians.

KEYWORDS: Argentina, Facebook, Social network, Technology appropriation, Community Libraries.

\section{INTRODUCCIÓN}

La adopción de Facebook por parte de las bibliotecas comenzó alrededor del año 2007, en principio en bibliotecas universitarias estadounidenses. No es casual, entonces, que la mayor producción de la literatura en el campo sobre la temática aborde usos en este tipo de bibliotecas.

Por otro lado, el paradigma de la Web 2.0 (Casey, 2006), que propone la construcción de servicios basados en las herramientas de la web social, el axioma "estar donde los usuarios están" (Margaix Arnal, 2008), junto con el éxito comercial que obtuvo la plataforma a nivel mundial por sobre otras redes sociales (Van Dijck, 2016), configuró a Facebook como tema de interés en el campo bibliotecario con una producción bibliográfica de fuerte impronta eficientista en la que predominan estudios que exploran principalmente la adopción de la herramienta en términos de extensión y de usos efectivos.

A la fecha y con una tradición de más de diez años de estudios que promueven la incorporación de Facebook y que proponen entre sus conclusiones sugerencias para usos efectivos, el arribo de las bibliotecas, 
a la plataforma, de acuerdo con los resultados de las investigaciones, se caracteriza como de llegada tardía, sin una planificación previa dentro de un plan de comunicación (Aquino, 2014, Laudano et al., 2014). Podemos decir en este sentido, que estamos frente a una literatura en la que predomina un tono prescriptivo sobre el que las bibliotecas parecen estar a la zaga.

Por otro lado, en relación al tema de este artículo, las características puntuales de la biblioteca popular en Argentina (Planas, 2014) hace que sea difícil trasladar los métodos y conclusiones obtenidos hasta el momento en estudios previos de bibliotecas universitarias, especializadas y aún públicas (Laudano et al, 2016). Atento a estas diferencias y particularidades se hace necesario problematizar los enfoques basados en la eficiencia y la eficacia que detentan una mirada centrada en las tecnologías antes que los usos que hacen los sujetos de acuerdo con sus condiciones materiales y objetivas, su contexto, incluso la trayectoria previa que tiene el espacio en relación a las TIC.

En relación a estas áreas de vacancia que presenta la literatura, este trabajo se inscribe en línea de investigación de la apropiación de tecnologías (Andrés, 2014; Lago Martínez et al, 2018) para conocer las posibilidades de acción que tienen los usuarios de la plataforma, ya no en términos de usos ideales o adecuados, sino de acuerdo a la interpretación y el sentido que le otorga el colectivo de bibliotecas populares platenses según sus necesidades y en un entorno socio histórico determinado. Para ello, a través del diálogo con los actores, se procura realizar una revisión de las principales conclusiones de la bibliografía que el campo bibliotecario ha producido hasta el momento.

Se trata en definitiva de una indagación en torno a las prácticas cotidianas de los y las bibliotecarias en Facebook, lo que Michel De Certeau (2006) llamó las mil maneras de "cazar furtivamente". Perseguimos entonces, un enfoque distinto al que propone la teoría bajo la noción de estrategia que subordina la decisión de los y las usuarias de la plataforma a un cálculo para usos óptimos y efectivos de la herramienta. En el ámbito de lo cotidiano nos encontramos con las "tácticas del débil", aquellos movimientos rápidos que cambian la organización del espacio en el terreno del otro a la vez que configuran un hacer y saber que escapa a lo preestablecido.

\section{MARCo TEÓRICo}

La apropiación de las tecnologías es una línea de investigación que se consolidó en los últimos años en el área de las ciencias sociales, heredera de las investigaciones que desarrollan autores de los Estudios Culturales ingleses. Uno de sus principales exponentes fue Edward Thompson. El autor nos dice que "la actividad 'apropiación' forma parte de un extendido proceso de autoaprendizaje a través del cual los individuos desarrollan un sentido de ellos mismos y de los otros, de su historia, de su lugar en el mundo y de los grupos sociales a los que pertenecen" (Thompson, 1998, p. 23).

Según Morales (2018) la apropiación como categoría se centra en los procesos de reflexividad de los sujetos y permite problematizar todo tipo de determinismo tecnológico. Configura una matriz de análisis que, desde una perspectiva sociocultural, piensa la manera en que los seres humanos incorporan las tecnologías a sus quehaceres cotidianos, tanto en su dimensión práctica como en su dimensión simbólica (Becerril Martínez, 2018).

Es concurrente con esta matriz de análisis la definición de Facebook como artefacto sociotécnico (Thomas y Buch, 2008), es decir, una tecnología se construye en los sentidos y los significados que le otorgan los y las usuarias en un proceso permanente de negociación.

Por último, como señala Andrés (2014), en relación a los trabajos de investigación, es necesario replantear los modelos imperantes en el estado del arte sobre el funcionamiento y el uso social extendido de las tecnologías. Estos modelos, como podrá verse en el apartado próximo de este trabajo, se encuentran presentes en las abundantes investigaciones que basan sus análisis en discursos del déficit y revelan, a su vez, una mirada 
tecnófila, toda vez que afirman que los sujetos se convierten en productores de contenidos por el simple hecho de disponer del acceso a las TICs.

\section{ANTECEDENTES}

Los primeros estudios sobre usos de Facebook en bibliotecas datan del año 2007 (Charnigo y BarnettEllis, 2007; Chu y Meulemans, 2008) y pertenecen al ámbito de las bibliotecas universitarias estadounidenses. A partir del año 2008 surgen los primeros trabajos en el ámbito español. Se puede considerar trabajo pionero en Argentina el de De Volder y Gutiérrez (2010) sobre bibliotecas universitarias.

En el ámbito de América Latina para usos de Facebook en bibliotecas públicas (tipo afín a la biblioteca popular por sus características), podemos citar a Mendes, Da Silva y Lópes Alves (2015) para usos de Facebook en bibliotecas de Santa Catarina; Bernardino Rodríguez, Suaiden y Cuevas Cerveró (2014), quienes exploraron los usos de Facebook por parte de bibliotecas públicas en el estado de Ceará; Calil y Almendra (2016) enfocados en las posibilidades de Facebook en el marco de programas de alfabetización informacional.

En los artículos relevados se presentan dos grandes objetivos de investigación: por un lado, los estudios buscan dar cuenta de la visibilidad o presencia de las bibliotecas de un tipo o región en Facebook; por otro, los artículos que se proponen describir los usos. Dentro del primer grupo predominan los análisis de tipo cuantitativo. La presencia se mide en términos de números absolutos, de cantidad de páginas por región o por tipo de biblioteca, por lo general sin discriminar entre cuentas activas o en desuso. Dentro del segundo grupo predomina el conteo de publicaciones e interacciones considerando resultados positivos o negativos en relación a cantidad de población real y potencial de las bibliotecas (Aquino, 2014). En términos generales los estudios arrojan valores muy bajos de uso por parte de las bibliotecas de la región.

Son escasos los trabajos que indagan sobre las percepciones de uso de los y las bibliotecarias. En Argentina, Cortés y Dugatto (2013) entrevistaron a los bibliotecarios del staff de la Biblioteca Central de la Universidad de Córdoba, así como también a los estudiantes, para indagar por los usos y consumos de nuevos medios en la comunidad de pertenencia de la biblioteca.

Con respecto a trabajos puntuales sobre Facebook en bibliotecas populares, existe un solo estudio que explora los usos en las bibliotecas de La Plata, Berisso y Ensenada. Se trata del trabajo de Laudano et al. (2016). Los autores señalan para el conjunto analizado, algunos puntos de coincidencia con otros tipos de bibliotecas: la llegada tardía de las bibliotecas populares a la red social, que para el conjunto estudiado es el año 2013 y la baja y muchas veces irregular frecuencia de posteos. Los autores muestran, también, los límites que presenta la observación de las páginas a la hora de explicar estos resultados y el comportamiento atípico de uno de los trabajos del estudio. Entre sus recomendaciones proponen recurrir a otras metodologías de estudio que apunten a explorar las circunstancias materiales objetivas en las que tienen lugar los modos en que las bibliotecas hacen usos diferenciales de Facebook (Laudano et al., 2016). De esta manera, el estudio deja abierto el camino para profundizar el análisis sobre el uso y la apropiación de Facebook en bibliotecas populares; ya que las exploraciones de corte cuantitativo por el momento parecen no agotar las preguntas.

\section{Metodología}

De acuerdo con la categoría apropiación de tecnologías (Andrés, 2014), este trabajo prioriza la perspectiva del bibliotecario, no sólo para dar cuenta de las características que adquiere el uso de Facebook en las bibliotecas populares del Gran La Plata (Argentina). Se consideran miradas, perspectivas y marcos de referencia alrededor de los cuales los actores organizan sus prácticas. 
Para cumplir este objetivo se recurre a entrevistas semiestructuradas a responsables de bibliotecas. La entrevista constituye una modalidad de conversación (De Sena et al., 2012) y requiere como momento previo un trabajo exhaustivo de búsqueda de información, una "inmersión" por parte del entrevistador para conocer los mundos posibles desde los cuales los entrevistados se expresarán. Los temas ordenadores que conforman el guión de la entrevista surgen del conjunto de hipótesis teóricas que reúne la bibliografía del campo disciplinar y de un trabajo previo de observación de perfiles y páginas de Facebook de las bibliotecas populares que siguió la metodología de Laudano et al. (2016).

Es así que sobre 30 cuentas de Facebook que cuentan con un perfil activo, es decir, que presentaron al menos una publicación en los últimos dos meses del año 2016 se seleccionaron los casos para realizar las entrevistas. Para la muestra no intencional se tuvieron en cuenta perfiles y páginas de Facebook de bibliotecas de la región con una antigüedad de dos o más años. El conjunto seleccionado compone un grupo heterogéneo en cuanto a ubicación territorial y pertenencia institucional. Una de ellas pertenece al casco urbano y las otras tres a barrios periféricos: Villa Elisa, Tolosa y Los Hornos. Tienen, además, distintas trayectorias: una lleva 100 años de actividad ininterrumpida; las otras dos han tenido diferentes cierres a lo largo de los años. La cuarta tiene 20 años de antigüedad y lleva varias mudanzas. Son distintas, también, sus inserciones institucionales: una biblioteca pertenece a un club, otra a un círculo social y dos fueron creadas por asociaciones vecinales. En todos los casos, las bibliotecas reciben subsidio de la Comisión Nacional de Bibliotecas Populares (CONABIP) y de provincia de Buenos Aires -Argentina-. Además, se trata de bibliotecas gestionadas por profesionales que cursaron estudios superiores en el área de bibliotecología: en tres de las bibliotecas entrevistadas se combina el trabajo de recursos de formación técnica superior y recursos de formación universitaria.

Las entrevistas presenciales, que tuvieron un promedio de una hora de duración, se realizaron durante el mes de agosto de 2016 en cuatro bibliotecas. Entre las personas entrevistadas se encuentran: tres bibliotecarias, un bibliotecario y una presidenta de biblioteca, quien participó de manera activa en una de las entrevistas. ${ }^{1}$

\section{ANÁLISIS Y DISCUSIÓN}

En este apartado se procede al análisis de las entrevistas con la transcripción de algunos fragmentos significativos. Organizamos este análisis alrededor de tres núcleos que permiten establecer un contraste entre aquello que relatan los actores y las principales conclusiones de la bibliografía del campo. El primer tópico refiere a las razones por las que las bibliotecas deben adoptar Facebook. De acuerdo con la bibliografía, el objetivo principal para incorporar la herramienta es lograr mayor visibilidad en la red. Un segundo eje indaga por los aspectos relacionados con la gestión de la herramienta en sus dimensiones concretas: los recursos humanos, tecnológicos, tiempos y espacios. Por último, abordamos el tema de la planificación de las publicaciones y la selección de contenido.

\section{FACEBOOK EN LA BIBLIOTECA POPULAR: ¿VIRTUALIZACIÓN DE SERVICIOS O VISIBILIDAD EN} LA RED?

La primera constatación que puede hacerse es que el uso de las redes sociales no implica un proceso de digitalización ni un pasaje o transición a un nuevo tipo de biblioteca como supo proponer gran parte de la literatura promocional de la Web 2.0 analizada en el apartado de antecedentes de este trabajo (Aquino, 2014; Mendes Mendes, Garcês da Silva y Lopes Alves, 2015). La adopción de Facebook por parte de las bibliotecas no significa en sí misma un cambio radical de los procesos de trabajo o de la concepción de los servicios, aunque no se puede negar que se han intensificado y acelerado algunos de estos procesos. 
Los bibliotecarios no perciben estar frente a un cambio de paradigma, aun cuando reconocen las potencialidades que brindan estas herramientas. No existe una conciencia ni voluntad previa de incorporar las redes en pos de convertirse en una biblioteca 2.0. Es así que, aunque las bibliotecas tienen un blog donde publican contenidos con cierta periodicidad, asocian como antecedente de Facebook al teléfono en tanto permitía una comunicación "inmediata" con los usuarios. Los y las bibliotecarias refieren que en períodos previos al uso de Facebook respondían consultas por teléfono sobre cuestiones como horarios o disponibilidad de libros. Esta comunicación se traslada luego al chat del Facebook por su "gratuidad" e inmediatez, y sirvió incluso como medio de comunicación con los usuarios y entre el propio personal.

Por otro lado, las bibliotecas populares están atravesadas por la cultura tipográfica -en un sentido "macluhiano"- y sobre el impreso se asientan gran parte de su gestión administrativa y sus prácticas de difusión: la presencia de folletos, volantes, almanaques, formularios, carteles y carteleras son frecuentes en los escritorios de trabajo y en las salas de lectura como medio de difusión y comunicación. Esto se pudo constatar en las entrevistas a través de fotografías de los espacios y la observación de situaciones en las que la bibliotecaria o el bibliotecario ofrece un folleto de difusión o un formulario de asociación a los potenciales socios.

En el curso de las entrevistas y de manera no esperada se dieron dos constataciones importantes. La primera de ella se relaciona con la fecha de creación de las cuentas, según la bibliografía consultada, el año promedio de ingreso de las bibliotecas populares a la plataforma es el año 2013 (Laudano et al., 2016). La noción de "llegada tardía" había sido señalada también por De Volder y Gutiérrez para las bibliotecas universitarias en 2010 y por Laudano, Corda, Planas y Kessler en 2014 para las bibliotecas de centros de investigación. A partir de las entrevistas se puede concluir que es difícil estimar el año de creación de las cuentas de las bibliotecas populares. La falta de precisión de este dato se justifica por la rotación de personal, característica usual de las bibliotecas populares. La rotación de personal en la biblioteca popular es un factor que dificulta otros procesos de implementación de tecnologías en estos espacios como lo señalaron Dos Santos y Ruiz (2016) para la experiencia de implementación del software de gestión bibliotecaria DiGIBePe.

Los entrevistados no pueden dar fecha precisa del momento en que su biblioteca creó la cuenta de Facebook y para los casos analizados esa fecha, sería entre cinco y seis años anterior a la que se pudo establecer en el registro de la información que ofrece la plataforma. De este modo, el año estimado de creación de las cuentas de Facebook sería el año 2010.

Una de las bibliotecas centenarias de la ciudad: su presidenta y la bibliotecaria de la institución, cuando se les pregunta desde cuándo utiliza Facebook la biblioteca, dicen que ésta es su tercera cuenta:

Entrevistadora (E): No sabía que tenían ese problema. ¿Llegan a una cantidad de amigos y ustedes tienen que generar otra cuenta?

Presidenta de biblioteca (P): Creo que es el tercero éste...El primero fue Biblioteca Popular A. I., el segundo Biblioteca A.

I. y este A. I. (risas). El otro día yo lo comentaba esto y se me ocurrió: Nos queda, A. I. Biblioteca o A. I....

E: ¿Porque no les deja poner el mismo nombre de vuelta?

Biblioteca 2 (B2): Se da cuenta, si vos pones biblioteca, como es una asociación, Facebook se da cuenta que es una organización...

P: Es muy perverso Facebook (risas)

B2: Esas palabras ahora no te las permite si vos querés crear ahora, o tenés que usar esas palabras, tenés que ponerlo como si fueras una persona.

En el caso de las bibliotecas populares el pasaje obligado del perfil a la página forma parte del problema del nombre de las instituciones. La falta de normalización en los nombres utilizados por las bibliotecas dificulta su identificación en búsquedas en internet (De Volder y Gutiérrez, 2010; Laudano, Corda, Planas y Kessler, 2014; Laudano et al., 2016) y su comunidad se circunscribe a aquellos usuarios de internet que conocen su identidad por lo que la "visibilidad" y la presencia se ven afectadas. Que en la modalidad perfil no puedan utilizar la palabra biblioteca o que la creación de varias cuentas no permitiera reutilizar la denominación hizo que los bibliotecarios debieran recurrir a estrategias acortando o invirtiendo las palabras que componen el nombre, generando, además, juegos de sentido con los usuarios. 
Biblioteca 1 (B1): Sí, porque en un principio estaba solamente el perfil, pero Facebook obligaba a que, si sos una institución, digamos, no tenía que tener el nombre de la institución tenía que ser una persona y que creemos la página. Así que se creó la página en 2015 y el perfil se le cambió el nombre como si fuera una persona, Juan V. (risas) Que de hecho me pasa que cuando escribo: "che, Juan" (risas). No sé fue una decisión en ese momento, de hecho ahora no me permiten el cambio de nombre, yo intenté cambiar el nombre por ejemplo, Juan V. Biblioteca y ya no me lo permiten porque se cambió el nombre varias veces.

Las bibliotecas escogidas para las entrevistas se dividen entre aquellas que utilizan la modalidad perfil y las que emplean la modalidad página, aunque si se tiene en cuenta que una de las bibliotecas tiene perfil y página actualizados, y otra ha decidido mantener su perfil, podemos advertir que existe una preferencia de las bibliotecas por esta última modalidad. La bibliografía especializada recomienda el uso de la modalidad página argumentando que ésta, por sobre el perfil, es más eficaz a la hora de lograr presencia en la red (Aquino, 2014). Los entrevistados, por otra parte, refieren que el pasaje a la modalidad página no fue una elección, sino una acción a la que los “obligó" la empresa. Asimismo, destacan que les resultaba más cómodo y práctico el perfil para la comunicación con los usuarios:

B1: En un principio, en 2015, íbamos a mantener la página y el perfil, es solo el perfil. Para no confundir. Pero después, lo que yo veía, lo empecé a hacer hace dos o tres meses atrás, podía tener más interacción, mediante el perfil que mediante la página, en algunas cosas.

E: ¿Cómo en qué?

B1: Sobre todo en los mensajes, en la forma de comentar en las publicaciones de los demás y eso. O sea, utilizarlo como si fuera una persona, como si la biblioteca fuera una persona, en el sentido, no solo una institución. Lo que hago: comparto lo de la página lo comparto también en la biografía porque veía que muchas personas no miraban. Miraban la página pero siempre se remitían al perfil, no sé por qué, quizás por la costumbre o es lo primero que les aparecía, pero siempre lo que yo publicaba en el perfil les parecía más visible...

El uso del perfil para la comunicación no se limita sólo al contacto con los usuarios pues también parece resultar más útil para la comunicación entre los integrantes de la biblioteca:

B2: Claro, es que lo que pasa es que antes no podías comunicarte, nos comunicamos mucho con los socios por mensaje privado. Te preguntan bueno, tal libro y esas cosas... pero ahora me (...) inclusive para nosotros nos servía mucho porque no te salía ningún costo y por privado te comunicabas inmediatamente.

Por otro lado, el perfil cuenta con herramientas que les resultan útiles para sus propias estrategias de difusión. Así explica un bibliotecario otra de las razones por las que prefiere el perfil a la página:

B1: Si, y además, no sé. ¿Sabés en dónde me entró la duda de por qué no usaba el perfil? ¿Viste? Veía que hacían cartelitos de colores con los fondos visibles ey porque yo no puedo hacerlo en la página? Y no está la herramienta esa, solamente puede hacerse de los perfiles. Entonces, ese tipo de cosas, parecen pavadas, pero es visual.

La preocupación de los bibliotecarios no se circunscribe a cuestiones de visibilidad o presencia en la red sino a la necesidad de contar con un medio de comunicación instantáneo y gratuito, con sus usuarios y usuarias o personal. Estas interacciones no pueden registrarse ni medirse desde la observación como usuario por lo que se matizan las conclusiones que refieren un uso estático de Facebook por parte de las bibliotecas.

Retomando la metáfora de De Certeau (2006) para el cierre de este apartado, aquello que identificamos como "sus propias estrategias", responden a ese movimiento rápido, un conjunto de tácticas que resuelve en el momento a través de intervenciones, situaciones que se presentan como desfavorables.

\section{LA GESTIÓN DE FACEBOOK: RECURSOS HUMANOS, TECNOLÓGICOS, TIEMPOS Y ESPACIOS}

Si bien no existe una planificación escrita o un acuerdo previo sobre los contenidos para publicar en el Facebook de la biblioteca, lo cierto es que la presencia de rutinas y un permanente trabajo de interpretación de la "respuesta" de los amigos/seguidores terminarán conformando en la práctica, un conjunto de criterios implícitos. 
Sobre el lugar desde el cual realizan las publicaciones, los y las entrevistadas manifiestan que les resulta incómodo utilizar el teléfono para gestionar las publicaciones de Facebook. Las publicaciones se realizan desde las PC de escritorio. En este sentido, existe una rutina que se repite en cada biblioteca: cuando se abre, se ingresa a la cuenta de Facebook que permanecerá abierta durante toda la jornada laboral. En esos primeros momentos, que según estiman los entrevistados les toma entre 40 minutos y una hora todos los días, revisan los mensajes y realizan las publicaciones. Sólo en el caso de la biblioteca que tiene horario reducido los entrevistados refieren que la gestión de Facebook se realiza exclusivamente durante las horas de trabajo. Sin embargo, cuando se indaga un poco más se puede ver que los márgenes del tiempo laboral son difusos. Una de las bibliotecarias relata que se encargó de publicar las efemérides durante sus vacaciones.

Sobre el tiempo que insume el uso de la herramienta, los entrevistados expresan rotundamente que no les conlleva demasiado. Durante la conversación, no obstante, puede verse que la gestión de Facebook lleva más tiempo y recursos de los que ellos mismos asignan en un principio. Esto ocurre, por ejemplo, al momento de responder las consultas de los usuarios. En todos los casos los entrevistados señalan que contar con un catálogo en línea podría reducir los pasos de la consulta de materiales que reciben por chat de Facebook. Las cuatro bibliotecas se encuentran actualmente en proceso de incorporar este catálogo en línea.

Responder los mensajes no es una tarea que se circunscribe a una respuesta de tipo "sí/no". Implica buscar el material en el catálogo y confirmar su disponibilidad para préstamo; es decir, se trata de una forma de servicio de referencia sincrónico (Borrell, 2008). Con las redes sociales, estos intercambios forman parte de las rutinas de usar Facebook. No hay un registro ni una medición de cantidad de pedidos por inbox ni tampoco la inscripción del servicio de referencia en línea como tal. De este modo, no se considera la incorporación de Facebook como una forma de virtualización de servicios tradicionales como el servicio de referencia.

La percepción de que las redes sociales pueden resultar una sobrecarga para las tareas es algo señalado por los responsables de medios de las bibliotecas de Córdoba (Dugatto y Cortes, 2013). Esta sobrecarga se presenta en la cantidad de consultas recibidas por chat que se han incrementado y por la dinámica de la red social que, según ellos entienden, "obliga" a responder de inmediato.

La gestión de Facebook conlleva más de un recurso humano. En los casos donde hay más de una persona a cargo, la gestión siempre es compartida, y aunque al principio parezca una distribución equitativa, puede verse luego que existen distribuciones de tiempo y tareas de acuerdo a cómo valora el equipo las distintas tecnologías que coexisten en el espacio. A veces uno de los integrantes de la biblioteca dedica más tiempo que el otro a la gestión de la plataforma. En estos casos existe una división, que asigna a una de las personas casi exclusivamente las tareas técnicas y a otra, más tiempo a la comunicación.

Según esta afirmación, la distribución de tareas en la biblioteca popular respondería al tipo de formación que poseen sus integrantes. A las bibliotecarias con formación universitaria se les asigna tareas relacionadas con los procesos técnicos, desarrollo y gestión de DiGiBePé, ya que se considera que portan saberes avanzados en cuestiones tecnológicas. Por su parte, los bibliotecarios de terciario se presentan como "exploradores" y "autodidactas" de tecnologías y son los responsables de la selección y gestión de las herramientas de comunicación. Las entrevistadas manifiestan que no recibieron ningún tipo de capacitación para el manejo de redes sociales que, por otro lado, no consideran necesaria. Interrogadas por capacitaciones para el personal de la biblioteca en materia de uso de redes sociales, las responsables del espacio las consideran innecesarias puesto que esos saberes los “portan” las bibliotecarias jóvenes por su condición de jóvenes o por su condición de profesionales universitarias. Esto se resume en frases del tipo "las chicas nacieron con las redes". Existe aquí una valoración que asigna distintos pesos simbólicos de las tecnologías. Esta valoración otorga una división de tareas en la que los responsables de la carga de registros en el catálogo quedan exclusivamente asignado a los recursos con formación en el área de bibliotecología, como se dijo más arriba, preferentemente de formación universitaria, mientras que las tareas relacionadas con la gestión de las redes puede ser realizada indistintamente y de manera compartida por bibliotecarios, directivos y voluntarios sin que tenga alguna incidencia el tipo de formación. 


\section{LAS PUBLICACIONES: CONSTRUCCIÓN, FUENTES, CRITERIOS Y SILENCIOS}

En este apartado recopilamos de las entrevistas algunas referencias que dan cuenta del modo que se construyen las publicaciones, modos que su vez, aunque no sean planificados responden a criterios que están internalizados en el quehacer cotidiano.

La frase que aparece con frecuencia en las entrevistas, "como si fuera una persona”, es una metáfora que explica además el lugar desde donde las bibliotecarias y bibliotecarios obtienen los contenidos para publicar en sus Facebook. Cuando se le pregunta a uno de los bibliotecarios por qué no recurre a la opción de publicaciones programadas que ofrece la plataforma como lo hacen con las entradas del blog que administran, explica y da las siguientes razones:

\section{E: ¿Y por qué no usás la programación?}

B1: ¿De publicaciones? No, porque se me ocurre en el momento la publicación. La hago. No la programo. Lo único que he programado es un evento, porque tenía tiempo en ese momento y ya lo dejé armado. Las hago en el momento o vengo con esa idea de que la voy a hacer, en esos minutos que te digo que le dedico, a chusmear las noticias, lo que está pasando en la comunidad (risas). Bueno, yo trato de participar en eso: hay un cumpleaños, "la biblioteca le desea feliz cumpleaños" (risas) y después me dedico yo a buscar algo, algo propio de la biblioteca y ahí lo dejo. Dejo el Facebook, a no ser que alguna otra cosa me vuelva a llevar, surge algo o, se me da en ese momento más tarde publicar. Bueno, ahí vuelvo al Facebook, lo tengo abierto y voy mirando.

Cuando se les preguntó si realizan algún tipo de planificación sobre los contenidos de Facebook, es decir si llegaron a algún tipo de acuerdo previo sobre el tipo de contenidos o si establecieron una periodicidad para las publicaciones, los entrevistados manifiestan que no existe una planificación.

Una de las bibliotecas al momento de la entrevista se encontraba atravesando su tercera mudanza física en tres años, sin PC ni conexión a internet y con horario de atención reducido. La bibliotecaria, única responsable del espacio, tiene una percepción negativa del uso que hace de Facebook su biblioteca y explica que la periodicidad de sus publicaciones se debe a que no tiene qué mostrar.

E: ¿Publicás una vez por semana, por ejemplo?

Biblioteca 3 (B3): Depende de lo que hay que hacer. Ahora que tenemos la semana de la lectura, van a venir unas narradoras (...) No comparto tantas cosas. Lamentablemente no tenemos la opción de decir: "icompramos tales libros!" (risas). Yo miro y veo la feria del libro... (risas) Compartís alguuuuuna cosa...

Cabe preguntarse desde esta perspectiva y frente a estas situaciones, si las publicaciones, su cantidad y periodicidad pueden responder a un criterio prefijado como propone la bibliografía relevada. Así, por ejemplo, algunos estudios (Aquino, 2014; Laudano, Corda, Planas y Kessler, 2016) proponen un aumento en la periodicidad de las publicaciones como estrategia comunicativa para lograr mayor visibilidad. Sin embargo, para los bibliotecarios los posteos y sus interacciones responden al ritmo de las actividades que desarrolla la biblioteca y a un sentido que se construye sobre lo que quieren decir en ese momento. En otras palabras, la estrategia de comunicación, según ellos, responde a los contenidos antes que a la frecuencia; prefieren resignar publicaciones si consideran que no hay qué decir. Los bibliotecarios manifiestan que las noticias que publican en algunos casos provienen de los grupos de Facebook que siguen sobre bibliotecología. De allí escogen noticias de divulgación relacionadas con el libro y las bibliotecas. Aquí opera una selección que filtra publicaciones que pueden no interesar a los amigos/seguidores por su complejidad y/o especificidad disciplinar. Asimismo, existe una permanente atención a lo que publican sus usuarios u otras bibliotecas y esta atención se extiende en horario no laborales.

Algunos contenidos son seleccionados desde sus cuentas personales, es decir en el tiempo en que atienden a su Facebook personal está operando en ellos la selección de contenidos para el Facebook de la biblioteca. De esta manera, la frontera que separa el tiempo de ocio del de trabajo se vuelve borrosa. 
B1: Eso me implica que lo que es redes sociales, es que yo llego a la biblioteca le destino más o menos 45, 50 minutos a revisar y también a ser chusma, me pongo a chusmear a la gente lo que me parece en el inicio y me pongo a chusmear a chusmear y tengo una idea de unos intereses y algunos comparten cosas interesantes también.

Tareas relacionadas con el monitoreo de las publicaciones como el seguimiento de la cantidad de me gusta, los vistos y alcance de los post en muchos casos constituye un punto de partida para definir la construcción de contenidos. Así en palabras de una de las bibliotecarias:

E: Claro, porque ustedes notaron que...

B4: Claro notamos que cuando la efeméride es de una o dos noticias... se lee, cuando la efeméride es muy larga y tienen como 10 noticias que vienen en el Google, por ejemplo, no la lee nadie.

E: ¿Y eso se dan cuenta por los me gusta o por la estadística de la página?

B4: No, porque tenemos el control de quien lee y quien no lo lee, "fue visto por tantas personas". Si no hubo interés no se pone más y listo.

Si se analiza que el criterio de selección positiva o negativa de los contenidos se construye atendiendo a la elección de contenidos ponderados en otras páginas de Facebook, es decir, contenidos que ya han sido puntuados positivamente, se puede inferir que opera en las publicaciones el funcionamiento que tienen Facebook o Google con sus algoritmos de recuperación de información y que presenta al usuario entre los primeros resultados aquellos que son más populares.

Por un lado los y las bibliotecarias refieren que hacen uso del perfil de Facebook "como si fuera una persona" y que la selección de contenidos para el muro no puede sustraerse del sujeto que en ese momento administra la cuenta. No se puede, dicen, dejar de lado los gustos personales, las percepciones, o, se podría agregar, lo que imaginan que sus amigos/seguidores esperan del Facebook de la biblioteca. Esta situación más que demostrar la falta de planificación, revela los procesos de interpretación y redefinición permanente que realizan estos sujetos de la herramienta. Según ellas y ellos las publicaciones no se planifican, sino que "surgen" en el momento sin que puedan advertir, no tienen que hacerlo, todo el trabajo de lectura y relectura, el permanente trabajo de selección y toma de decisiones que realizan en base a la información y las posibilidades que les brinda la plataforma, empezando por la cantidad de me gusta, los vistos obtenidos, pero también en una lectura extensa de lo que ellas y ellos ven que otros y otras dicen o no dicen sobre un tema en la red, de lo que otros y otras prefieren o no prefieren de una temática.

B3: ¡Justo, justo, hoy pensé en eso! Viste que está esto “¿Dónde está Santiago Maldonado?” 2 ... La única vez que compartí algo, porque obviamente, trato de no hacer ningún comentario, pero absolutamente nada que afecte la sensibilidad de la gente, la única vez que compartí algo fue por la marcha de las mujeres el año pasado y me pasó que alguien comento algo... algo, no me acuerdo que... si buscas la publicación está. Yo traté de contestarle, no sé, lo mejor posible, no sé, como decirle "pero la violencia está"... y bueno me pasa eso ahora, que veo que ponen Santiago Maldonado y hacen comentarios... si ponés... ¿ ¿cómo lo va recibir el otro? y si no ponés, van a pensar.... en el personal no lo he puesto tampoco...No lo vi en otras bibliotecas tampoco...

Esto tiene su correlato con observación de los muros de las bibliotecas que presenta temas preferidos para las publicaciones (Laudano et al., 2016). Cuantitativamente predominan los contenidos que refuerzan la cultura del libro y la difusión de saberes de divulgación bibliotecológica que, al mismo tiempo reciben mayor respuesta por parte de los usuarios. Aquello que no pertenece a la esfera del libro, la cultura y la biblioteca parece pertenecer a la dimensión de lo personal, de una subjetividad que debe ser censurada, según ellas y ellos explican, porque no se considera "común". En este caso como en el resto de los criterios que se pudieron reconstruir, no existe una condición que inscriba unos como aceptables o inaceptables, se ejerce la autocensura. Al mejor estilo Bartleby, el escribiente ${ }^{3}$, dicen “preferiría no hacerlo”. 


\section{Conclusiones}

El uso de tecnologías en las bibliotecas populares ha sido tema de escasa atención en la bibliografía del campo. Existen algunos artículos que evalúan el grado de desarrollo e implementación de los softwares de gestión de CONABIP y sólo un trabajo específico que explora los usos de Facebook en este tipo de bibliotecas. Esta situación dificulta la reconstrucción de la historia del proceso de implementación de tecnologías en la biblioteca popular que permitiría, entre otras cuestiones, la comprensión de las características que asume la adopción de Facebook en el presente. Este trabajo realiza su aporte sobre esta área de vacancia al indagar por los usos Facebook en las bibliotecas populares de la región.

La categoría de apropiación de tecnologías permitió un acercamiento a los usos de Facebook a partir de la combinación de distintas técnicas de investigación. La observación y las entrevistas resultaron instancias complementarias para la re lectura los resultados obtenidos en investigaciones previas, entre ellos la afirmación que otorga una llegada tardía a la plataforma o la escasa interacción con los usuarios.

Frente al uso normalizado que propone la bibliografía especializada se encuentran los usos singulares que hacen las bibliotecas populares de la plataforma. Si bien no se construyen sobre una planificación previa, es posible reconstruir que la utilización se asienta sobre criterios tácitos que revelan el modo en que las bibliotecas populares se apropian, interpretan y, en algunos casos, resignifican la plataforma.

La visibilidad y la conexión son tan importantes para las bibliotecas populares como para los otros tipos de bibliotecas, aunque la incorporación de Facebook en las bibliotecas populares se debe más que a una cuestión de visibilidad o de virtualización de servicios a la necesidad concreta de contar con un medio de comunicación gratuito e instantáneo, tanto para el intercambio con los usuarios como con el personal de la biblioteca.

Otro aporte que realiza el trabajo refiere a la disposición de recursos en el espacio biblioteca, principalmente en relación a sus recursos humanos. La división de tareas que se asienta sobre una división simbólica de las tecnologías que conviven en la biblioteca, que por otro lado no parecen converger en un objetivo preestablecido: la modernización de servicios.

En relación a las publicaciones, su periodicidad y contenido, se ven favorecidas en número aquellas publicaciones aparentemente "neutrales" que tiene mayor recurrencia, como es el caso de los posteos relacionados con los mensajes culturales. Es susceptible de futuras indagaciones ahondar en las retóricas que construyen las publicaciones sobre un hipotético modelo de biblioteca popular como "espacio universal", "espacio común".

Finalmente, la categoría de apropiación de las TIC, permitió una serie de descentramientos. El primero de ellos es el que desplazó el centro de atención de análisis basado en la herramienta y que por lo tanto circunscribe la comprensión de los usos desde el grado de adecuación del usuario al dispositivo. Un segundo descentramiento operó sobre la investigadora quien para este trabajo debió acercarse a los espacios de trabajo pero también, a través de la pregunta, a las subjetividades de los propios actores. Estos descentramientos parten de la incomodidad que surge de la brecha que advertimos entre las propuestas teóricas y lo que efectivamente ocurre en la práctica. Las tácticas del débil (De Certeau, 2006) que pudimos relevar en este trabajo, significan una aproximación a las prácticas cotidianas desde un enfoque científico y se presentan como una posibilidad rica en hallazgos susceptible de ser mejorada en futuras investigaciones.

\section{REFERENCIAS}

Andrés, G. D. (2014). Una aproximación conceptual a la "apropiación social” de TIC. Questión, 1(43). Recuperado de https://perio.unlp.edu.ar/ojs/index.php/question/article/view/2227/1963

Aquino, S. (2014). Análisis del uso de Facebook en las bibliotecas académicas peruanas. Redescubriendo la educación en la era del conocimiento. Ponencia presentada en VI Jornadas Nacionales de Bibliotecas Universitarias, Altamira, Lima, Perú. Recuperado de http://eprints.rclis.org/23207/1/SilvanaAquino-Altamira2014.pdf 
Becerril Martínez, W. (2018). Indagar las apropiaciones tecnológicas desde una perspectiva de género feminista. En S. Lago Martínez, A. Álvarez, M. Gendler, y A. Méndez (Eds). Acerca de la apropiación de tecnologias: teoría, estudios y debates. Ciudad Autónoma de Buenos Aires. Rada Tilly. Del Gato Gris. Recuperado de http://www.delgatogris.com.ar/wp-content/uploads/2018/10/Lago-Mart\%C3\%ADnez-et-al-Acerca-de-la -apropiaci\%C3\%B3n-de-tecnolog\%C3\%ADas.pdf

Bernardino Rodrigues, M., Suaiden, E., y Cuevas Cerveró, A. (2014). O uso do Facebook pelas bibliotecas públicas do estado do Ceará. InCID: Revista de Ciência da Informação e Documentação, 1(5). https://doi.org/10.11606 /issn.2178-2075.v5ilp112-123

Borrell, M. (2008). Servicio de referencia virtual por chat en bibliotecas universitarias (Tesis de grado). Universidad Nacional de La Plata. Recuperada de http://www.memoria.fahce.unlp.edu.ar/tesis/te.382/te.382.pdf

Calil, A. y Almendra, G. (2016). As apropriações do Facebook pelas bibliotecas públicas estaduais brasileiras. Em Questão, 22(1). Recuperado de https://doi.org/10.19132/1808-5245221.188-213

Casey, M. (2006). Library 2.0: Service for the Next-Generation Library. Library Journal, noviembre 1. Recuperado de https://goo.gl/zkRPQp

Charnigo, L., y Barnett-Ellis, P. (2007). Checking Out Facebook.com: The Impact of a Digital Trend on Academic Libraries. Information, technology and libraries, 26(1). https://doi.org/10.6017/ital.v26i1.3286

Chu, M., y Meulemans, Y. (2008). The Problems and Potential of MySpace and Facebook Usage in Academic Libraries. Internet Reference Services Quarterly, 13(1), 69-85.

Cortés, M.B. y Dugatto, V. P. (2013). Usos, prácticas y consumos culturales de nuevos medios: Biblioteca Mayor de la Universidad Nacional de Córdoba. Trabajo presentado en III Jornadas de Intercambio y Reflexión acerca de la Investigación en Bibliotecología. Universidad Nacional de La Plata, La Plata, Argentina. Recuperado de http s://goo.gl/BCnovX

De Certeau, M. (2006). La invención de lo cotidiano. México, Universidad Iberoamericana.

De Volder, C., y Gutiérrez, F. (2010). La (no) presencia de las bibliotecas universitarias en Facebook. Las redes sociales y las bibliotecas universitarias. Ponencia presentada en 8 a Jornadas de Bibliotecas Digitales Universitarias, Facultad de Medicina, Buenos Aires.

De Sena, A., Del Campo, N., Dettano, A.; García Acevedo, M., y Saez Valenzuela, M. (2012). La entrevista como un modo de indagación social. Una experiencia compartida. En G. Gómez Rojas y A. De Sena (comp.). En clave metodológica. Reflexiones y prácticas de la investigación social. Buenos Aires: Ediciones cooperativas. Recuperado de http://pdfhumanidades.com/sites/default/files/apuntes/De\%20Sena\%20y\%20otras_0.pdf

Dos Santos, P., y Ruiz, P. (2016). DigiBepé: implementación del sistema de gestión bibliotecaria en las bibliotecas populares de la Argentina y su integración en el Catálogo Colectivo de la CONABIP. Trabajo presentado en $48^{a}$ Reunión ABGRA. Asociación de Bibliotecarios Graduados de la República Argentina, Buenos Aires. Recuperado de https://goo.gl/sDHnmw

Lago Martínez, S., Álvarez, A., Gendler, M., y Méndez, A. (2018). Acerca de la apropiación de tecnologias: teoría, estudios y debates. Ciudad Autónoma de Buenos Aires: Rada Tilly, Del Gato Gris. Recuperado de http://www.delgatogris.com.ar/wp-content/uploads/2018/10/Lago-Mart\%C3\%ADnez-et-al-Acerca-de-la -apropiaci\%C3\%B3n-de-tecnolog\%C3\%ADas.pdf

Laudano, C. N., Corda, M. C., Planas, J., y Kessler, M. I. (2014). Los usos de la red social Facebook en las bibliotecas de institutos y centros de investigación en Argentina. Palabra Clave [La Plata], 4(1), 20-32. Recuperado de ht tps://www.palabraclave.fahce.unlp.edu.ar/article/view/PCv4n1a02

Laudano, C. N., Corda, M. C., Planas, J., y Kessler, M. I. (2016). Los usos de la red social Facebook por parte de bibliotecas universitarias argentinas: Reflexiones en torno a las dinámicas comunicativas en la Web 2.0. Revista Interamericana de Bibliotecología, 39(1), 23-37. Recuperado de https://doi.org/10.17533/udea.rib.v39n1a05

Laudano, C., Corda, M.C., Planas, J., Kessler, M.I., y Aracri, A. (2016). Presencia y usos de Facebook en las bibliotecas populares de La Plata, Berisso y Ensenada (Argentina). Información, cultura y sociedad, 35, 107-123. Recuperado de http://revistascientificas.filo.uba.ar/index.php/ICS/article/view/2743 
Margaix Arnal, D. (2008). Las bibliotecas universitarias y Facebook: cómo y por qué estar presentes. El profesional de la información, 17(6), 589-600. Recuperado de http://www.elprofesionaldelainformacion.com/contenidos/20 08/noviembre/02.pdf

Mendes, M., Garcês da Silva, F. y Lopes Alves, B. (2015). Utilização do Facebook pelas bibliotecas públicas municipais do Estado de Santa Catarina. Revista ACB, 20(1), 41-48. Recuperado de https://revista.acbsc.org.br/racb/arti cle/view/990

Morales, S. (2018). La apropiación de tecnologías. Ideas para un paradigma en construcción. En S. Lago Martínez, A. Álvarez, M. Gendler y A. Méndez (Eds). Acerca de la apropiación de tecnologias: teoria, estudios y debates. Ciudad Autónoma de Buenos Aires: Rada Tilly, Del Gato Gris. Recuperado de http://www.delgatogris.com.ar/wp-content/uploads/2018/10/Lago-Mart\%C3\%ADnez-et-al-Acerca-de-la -apropiaci\%C3\%B3n-de-tecnolog\%C3\%ADas.pdf

Planas, J. (2014). Libros, lectores y sociabilidades de lectura. Una historia de los orígenes de las bibliotecas populares en la Argentina. Buenos Aires: Ampersand.

Thomas, H., y Buch, A., (Eds.). (2008). Actos, actores y artefactos: sociología de la tecnología (1. ed). Bernal: Universidad Nacional de Quilmes.

Thompson, J.B. (1998). Los media y la modernidad: una teoría de los medios de comunicación. Buenos Aires: Paidós.

Van Dijck, J. (2016). La cultura de la conectividad: una historia critica de las redes sociales. Buenos Aires: Siglo XXI.

\section{Notas}

1 Para resguardar la identidad de las entrevistadas se utiliza la letra $B$ con unos subíndices para diferenciar a las 4 bibliotecas. En algunos casos se recurrió a pseudónimos Las intervenciones de la entrevistadora se marcan con la letra E. En el caso en el que participó la presidenta de la biblioteca, ésta es nominada en los fragmentos a través de la inicial $P$.

2 El caso Santiago Maldonado se refiere a la desaparición y muerte de Santiago Andrés Maldonado a partir del 1 de agosto de 2017 en Resistencia de Cushamen, en la provincia argentina del Chubut. La misma se produjo en el contexto de la represión de Gendarmería Nacional a una protesta de la comunidad mapuche Pu Lof.

3 Bartleby, el escribiente es un cuento del escritor estadounidense Herman Melville. 